\title{
Simple model of bouncing ball dynamics: displacement of the table assumed as quadratic function of time
}

\author{
Andrzej Okniński • Bogusław Radziszewski
}

Received: 16 December 2010 / Accepted: 15 April 2011 / Published online: 12 May 2011

(C) The Author(s) 2011. This article is published with open access at Springerlink.com

\begin{abstract}
Nonlinear dynamics of a bouncing ball moving in gravitational field and colliding with a moving limiter is considered. Displacement of the limiter is a quadratic function of time. Several dynamical modes, such as fixed points, 2-cycles, grazing and chaotic bands are studied analytically and numerically. It is shown that chaotic bands appear due to homoclinic structures created from unstable 2-cycles in a corner-type bifurcation.
\end{abstract}

Keywords Nonsmooth dynamics · Bouncing ball . Exact solutions

\section{Introduction}

Vibro-impacting systems belong to a very interesting and important class of nonsmooth and nonlinear dynamical systems [1-4] with important technological applications [5-8]. Dynamics of such systems can be extremely complicated due to velocity discontinuity arising upon impacts. A very characteristic feature of such systems is the presence of nonstandard bifurcations such as border-collisions and grazing impacts which often lead to complex chaotic motions.

\footnotetext{
A. Okniński $(\varangle) \cdot$ B. Radziszewski

Politechnika Świętokrzyska, Al. 1000-lecia PP 7,

25-314 Kielce, Poland

e-mail: fizao@tu.kielce.pl

B. Radziszewski

e-mail: boradzi@tu.kielce.pl
}

The Poincaré map, describing evolution from an impact to the next impact, is a natural tool to study vibro-impacting systems. The main difficulty with investigating impacting systems is in finding instant of the next impact, what typically involves solving a nonlinear equation. However, the problem can be simplified in the case of a bouncing ball dynamics assuming a special motion of the limiter. Bouncing ball models have been extensively studied; see [9] and references therein. As a motivation that inspired this work, we mention the study of physics and transport of granular matter [6]. A similar model has been also used to describe the motion of railway bogies [7]. In such systems chattering and chaotic dynamics arise typically; see $[10,11]$ for a theoretical analysis.

Recently, we have considered several models of motion of a material point in a gravitational field colliding with a limiter moving with piecewise constant velocity [12-15]. Moreover, we have proposed more realistic yet still simple models approximating sinusoidal motion of the table as exactly as possible but still preserving possibility of analytical computations [16]. In the present work, we study the model in which displacement of the table is a quadratic and periodic function of time.

The paper is organized as follows. In Sect. 2 a onedimensional dynamic of a ball moving in a gravitational field and colliding with a table is reviewed and the corresponding Poincare map is constructed. A bifurcation diagram is computed for displacement of the table assumed as a quadratic and periodic function of 
time. In the next section, dynamical modes shown in the bifurcation diagram such as fixed points, 2-cycles, grazing and chaotic bands are studied analytically and numerically. It is shown that chaotic bands appear due to homoclinic structures created from unstable 2cycles in a corner-type bifurcation. We summarize our results in Sect. 4.

\section{Bouncing ball: a simple motion of the table}

We consider a motion of a small ball moving vertically in a gravitational field and colliding with a moving table, representing unilateral constraints. The ball is treated as a material point while the limiter's mass is assumed so large that its motion is not affected by impacts. A motion of the ball between impacts is described by the Newton's second law:

$m \ddot{x}=-m g$,

where $\dot{x}=d x / d t$ and motion of the limiter is

$y=y(t)$,

with a known function $y$. We shall also assume that $y$ is a continuous function of time. Impacts are modeled as follows:

$x\left(\tau_{i}\right)=y\left(\tau_{i}\right)$,

$\dot{x}\left(\tau_{i}^{+}\right)-\dot{y}\left(\tau_{i}\right)=-R\left(\dot{x}\left(\tau_{i}^{-}\right)-\dot{y}\left(\tau_{i}\right)\right)$,

where duration of an impact is neglected with respect to time of motion between impacts. In (3), (4), $\tau_{i}$ stands for time of the $i$ th impact, while $\dot{x}_{i}^{-}, \dot{x}_{i}^{+}$are left-sided and right-sided limits of $\dot{x}_{i}(t)$ for $t \rightarrow \tau_{i}$, respectively, and $R$ is the coefficient of restitution, $0 \leq R<1$ [5].

Solving (1) and applying impact conditions (3), (4), we derive the Poincaré map in nondimensional variables:

$$
\begin{aligned}
& \gamma Y\left(T_{i+1}\right)=\gamma Y\left(T_{i}\right)-\Delta_{i+1}^{2}+\Delta_{i+1} V_{i}, \\
& V_{i+1}=-R V_{i}+2 R \Delta_{i+1}+(1+R) \gamma \dot{Y}\left(T_{i+1}\right),
\end{aligned}
$$

where $\Delta_{i+1} \equiv T_{i+1}-T_{i}, Y(T)$ determines position of the table at time $T, V$ is velocity of the ball and $\gamma$ is a parameter depending on space and time scales and the acceleration $g$ [17]; see also [18] where analogous map was derived earlier. The limiter's motion has been typically assumed in form $Y_{S}(T)=\sin (T)$, cf. [13] and references therein. This choice leads to serious difficulties in solving the first of (5) for $T_{i+1}$, thus making analytical investigations of dynamics hardly possible. Accordingly, we have decided to simplify the limiter's periodic motion to make (5a) solvable.

In our previous papers, we have assumed displacement of the table as piecewise linear periodic function of time [12-15]. In our recent work, preliminary results for function $Y(T)$ assumed as quadratic, $Y_{q}$, and two cubic functions of time, $Y_{c_{1}}$ and $Y_{c_{2}}$, have been obtained [16]. In this work, we study dynamics for quadratic function of time $Y_{q}(T)$ :

$Y_{q}(T)= \begin{cases}-16 \hat{T}\left(\hat{T}-\frac{1}{2}\right), & 0 \leq \hat{T}<\frac{1}{2}, \\ 16\left(\hat{T}-\frac{1}{2}\right)(\hat{T}-1), & \frac{1}{2} \leq \hat{T} \leq 1,\end{cases}$

with $\hat{T}=T-\lfloor T\rfloor$, where $\lfloor x\rfloor$ is the floor functionthe largest integer less than or equal to $x$, cf. Fig. 1 where displacement of the table in the case of sinusoidal motion $Y_{S}(T)=\sin (2 \pi T)$ has been also shown.

Since the period of motion of the limiter is equal to one, the map (5) is invariant under the translation $T_{i} \rightarrow T_{i}+1$. Accordingly, all impact times $T_{i}$ can be reduced to the unit interval $[0,1]$. The model consists thus of (5), (6) with control parameters $R, \gamma$.

\section{Analytical and numerical results}

In Fig. 2 below, we show the bifurcation diagram with times of impacts computed for growing $\gamma$ and $R=$ 0.85 (see also [16] where bifurcation diagram with velocities just after impacts against $\gamma$ was shown). It follows that dynamical system (5), (6) has several attractors: six fixed points, one 2-cycle and, possibly, chaotic attractor.

We shall investigate now fixed points, 2-cycle and chaotic bands shown in Fig. 2, as well as grazing, combining analytical and numerical approach. General analytical conditions for birth of new modes of motion were given in [19].

\subsection{Fixed points and their stability}

We shall first study periodic solutions with one impact per $k$ periods. Such states have to fulfill the following 
Fig. 1 Displacement of the table: $Y_{q}(T)$-solid line, and $Y_{S}(T)$-dashed line

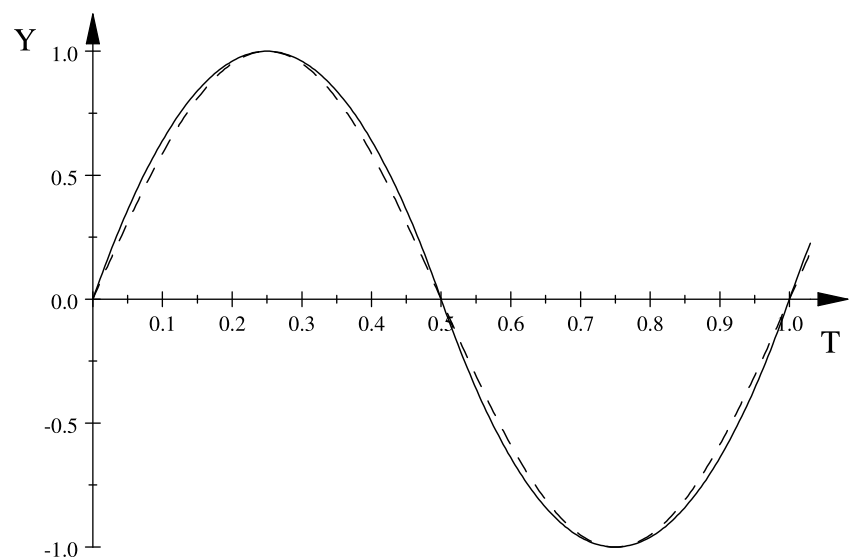

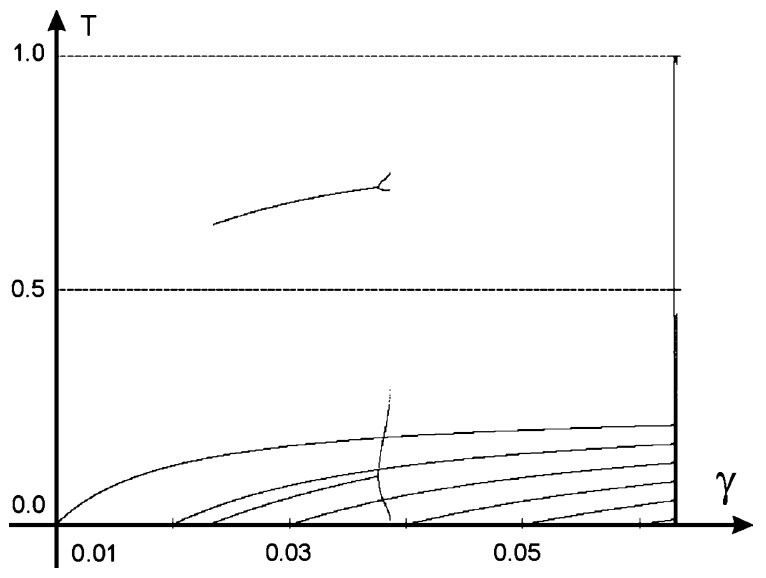

Fig. 2 Bifurcation diagram. $R=0.85, \gamma \in[0.0100,0.0635]$. Vertical critical line marks end of stability of fixed points and birth of chaotic dynamics

conditions:

$$
\begin{aligned}
V_{n+1} & =V_{n} \equiv V_{*}^{(k / 1)}, \quad T_{n+1}=T_{n}+k \equiv T_{*}^{(k / 1)}+k \\
(k & =1,2, \ldots),
\end{aligned}
$$

where

$T_{*}^{(k / 1)} \in(0,1), \quad V_{*}^{(k / 1)}>\gamma \dot{Y}_{q}(T)$.

Substituting these conditions into (5), (6), we obtain two sets of fixed points:

$0 \leq T_{* s}^{(k / 1)}=\frac{1}{4}-\frac{k(1-R)}{32 \gamma(1+R)} \leq \frac{1}{2}$,

$V_{* s}^{(k / 1)}=k$, where the impact occurs in time interval $T_{* s}^{(k / 1)} \in$ $\left(0, \frac{1}{2}\right)$ and

$\frac{1}{2} \leq T_{* u}^{(k / 1)}=\frac{3}{4}+\frac{k(1-R)}{32 \gamma(1+R)} \leq 1$,

$V_{*}^{(k / 1)}=k$

with impacts taking place in time interval $T_{* u}^{(k / 1)} \in$ $\left(\frac{1}{2}, 1\right)$.

Solutions (9) fulfill physical requirements and are stable in the following interval of $\gamma$ :

$k \frac{1-R}{8(1+R)} \leq \gamma \leq \frac{1+R^{2}}{8(1+R)^{2}}$,

where lower bound is a consequence of $T_{* s}^{(k / 1)} \geq 0$ while the upper bound follows from the condition that eigenvalues $\lambda$ of the stability matrix obey $|\lambda|<1$. On the other hand, solutions (10) are always unstable and are physical for

$\gamma \geq k \frac{1-R}{8(1+R)}$,

what is equivalent to the condition $T_{* u}^{(k / 1)} \leq 1$.

\subsection{Birth of the stable 2-cycle}

It follows from the bifurcation diagram, Fig. 2, that there exists a stable 2-cycle with time of the first impact $T_{* 1} \in\left(0, \frac{1}{2}\right)$ and time of the second impact $T_{* 2} \in$ $\left(\frac{1}{2}, 1\right)$. Such periodic solution must fulfill the following equations which are easily obtained from (5), (6): 


$$
\begin{aligned}
16 \gamma( & \left.T_{* 2}-\frac{1}{2}\right)\left(T_{* 2}-1\right) \\
= & -16 \gamma T_{* 1}\left(T_{* 1}-\frac{1}{2}\right)-\left(T_{* 2}-T_{* 1}\right)^{2} \\
& +\left(T_{* 2}-T_{* 1}\right) V_{* 1}, \\
V_{* 2}= & -R V_{* 1}+2 R\left(T_{* 2}-T_{* 1}\right) \\
& +\gamma(1+R)\left(32 T_{* 2}-24\right), \\
-16 \gamma & \left(T_{* 3}-1\right)\left(\left(T_{* 3}-1\right)-\frac{1}{2}\right) \\
= & 16 \gamma\left(T_{* 2}-\frac{1}{2}\right)\left(T_{* 2}-1\right)-\left(T_{* 3}-T_{* 2}\right)^{2}
\end{aligned}
$$

$$
\begin{aligned}
& +\left(T_{* 3}-T_{* 2}\right) V_{* 2}, \\
V_{* 3}= & -R V_{* 2}+2 R\left(T_{* 3}-T_{* 2}\right) \\
& +\gamma(1+R)\left(8-32\left(T_{* 3}-1\right)\right), \\
T_{* 3}= & T_{* 1}+1, \\
V_{* 3}= & V_{* 1} .
\end{aligned}
$$

Eliminating variables, we arrive at equation for time of the first impact only:

$$
C_{4} x^{4}+C_{3} x^{3}+C_{2} x^{2}+C_{1} x+C_{0}=0,
$$

where $x \equiv T_{* 1}$ and

$$
\begin{aligned}
& C_{0}=\frac{\left(8\left(R^{2}-1\right) \gamma+R^{2}+1\right)\left(128(R+1)^{3} \gamma^{2}+8\left(R^{2}-1\right)(3 R+1) \gamma+(R-1)\left(R^{2}+1\right)\right)}{(R+1)^{3}(R-1)^{2}}, \\
& C_{1}=-64 \gamma \frac{128\left(R^{2}-2 R-1\right)(R+1)^{2} \gamma^{2}+8(R+1)\left(3 R^{3}-3 R^{2}+7 R+1\right) \gamma+\left(R^{2}+1\right)(R-1)^{2}}{(R-1)^{2}(R+1)^{2}}, \\
& C_{2}=-2048 \gamma^{2} \frac{48 R(R+1) \gamma-R^{3}-R^{2}-5 R+1}{(R-1)^{2}(R+1)}, \\
& C_{3}=4096 \gamma^{2}(-1+16 \gamma) \frac{R^{2}+2 R-1}{(R-1)^{2}}, \\
& C_{4}=-4096 \gamma^{2}(-1+16 \gamma) \frac{R+1}{R-1} .
\end{aligned}
$$

It follows from the bifurcation diagram that the 2cycle is born when $T_{* 1}=x=0$. This in turn occurs when $C_{0}=0$. Equation $C_{0}=0$ has three roots:

$$
\begin{aligned}
\gamma_{1}= & \frac{1}{8} \frac{1+R^{2}}{1-R^{2}}, \\
\gamma_{2}= & \frac{1}{32(R+1)} \\
& \times \frac{-3 R^{2}+2 R+1-\sqrt{R^{4}-12 R^{3}-2 R^{2}+4 R+9}}{R+1}, \\
\gamma_{3}= & \frac{1}{32(R+1)} \\
& \times \frac{-3 R^{2}+2 R+1+\sqrt{R^{4}-12 R^{3}-2 R^{2}+4 R+9}}{R+1} .
\end{aligned}
$$

Testing (16) against numerical computations, we find out that the stable 2-cycle is born at $\gamma=\gamma_{3}$. For example, for $R=0.85$, we have $\gamma_{3}=0.0233674$, cf. Fig. 2.

3.3 $N$ impacts in the subinterval $0 \leq T<\frac{1}{2}$ or $\frac{1}{2} \leq T \leq 1$ and grazing

Let us assume that the first and the next impact start and end in the interval $0 \leq T<\frac{1}{2}$ or in $\frac{1}{2} \leq T \leq 1$. In these cases, equation for the time of the next impact (5a) has trivial solution $T_{i+1}=T_{i}$. We have from (5a) and (6): 


$$
\begin{aligned}
& -\gamma 16\left(T_{i}+\Delta_{i}\right)\left(T_{i}+\Delta_{i}-\frac{1}{2}\right) \\
& \quad+\gamma 16 T_{i}\left(T_{i}-\frac{1}{2}\right)=-\Delta_{i}^{2}+\Delta_{i} V_{i}, \\
& \gamma 16\left(T_{i}+\Delta_{i}-\frac{1}{2}\right)\left(T_{i}+\Delta_{i}-1\right) \\
& \quad-\gamma 16\left(T_{i}-\frac{1}{2}\right)\left(T_{i}-1\right)=-\Delta_{i}^{2}+\Delta_{i} V_{i},
\end{aligned}
$$

where $T_{i}, T_{i+1} \in\left[0, \frac{1}{2}\right], T_{i}, T_{i+1} \in\left[\frac{1}{2}, 1\right]$, respectively, and $\Delta_{i}=T_{i+1}-T_{i}$.

Nonzero solutions read

$$
\begin{aligned}
\Delta_{i} & =\frac{8 \gamma\left(4 T_{i}-1\right)+V_{i}}{1-16 \gamma} \\
0 & \leq \gamma<\frac{1}{16}, T_{i}, T_{i+1} \in\left[\frac{1}{2}, 1\right] \\
\Delta_{i} & =-\frac{32 \gamma T_{i}-24 \gamma-V_{i}}{16 \gamma+1}, \quad 0 \leq \gamma, T_{i}, T_{i+1} \in\left[\frac{1}{2}, 1\right]
\end{aligned}
$$

and can be written in terms of post impact relative velocity $v_{i} \stackrel{\text { df }}{=} V_{i}-\gamma \dot{Y}_{q}$ as

$$
\begin{aligned}
\Delta_{i} & =\frac{1}{1-16 \gamma} v_{i}, \\
0 & \leq \gamma<\frac{1}{16}, T_{i}, T_{i+1} \in\left[0, \frac{1}{2}\right], \\
\Delta_{i} & =\frac{1}{1+16 \gamma} v_{i}, \quad 0 \leq \gamma, T_{i}, T_{i+1} \in\left[\frac{1}{2}, 1\right],
\end{aligned}
$$

where $\dot{Y}_{q}(T)=-32 T+8$ for $0 \leq T<\frac{1}{2}$ and $\dot{Y}_{q}(T)=$ $-32 T+8$ for $0 \leq T<\frac{1}{2}$, cf. (6).

Finally, we obtain from (5b) in both cases:

$$
v_{i+1}=R v_{i}, \quad T_{i}, T_{i+1} \in\left[0, \frac{1}{2}\right] \text { or } T_{i}, T_{i+1} \in\left[\frac{1}{2}, 1\right] .
$$

We demand now that $T_{0}+\sum_{i=0}^{n-1} \Delta_{i}$ stays in the appropriate interval-this guarantees $n$ impacts in the appropriate interval. Accordingly, we get from (19) and (20):

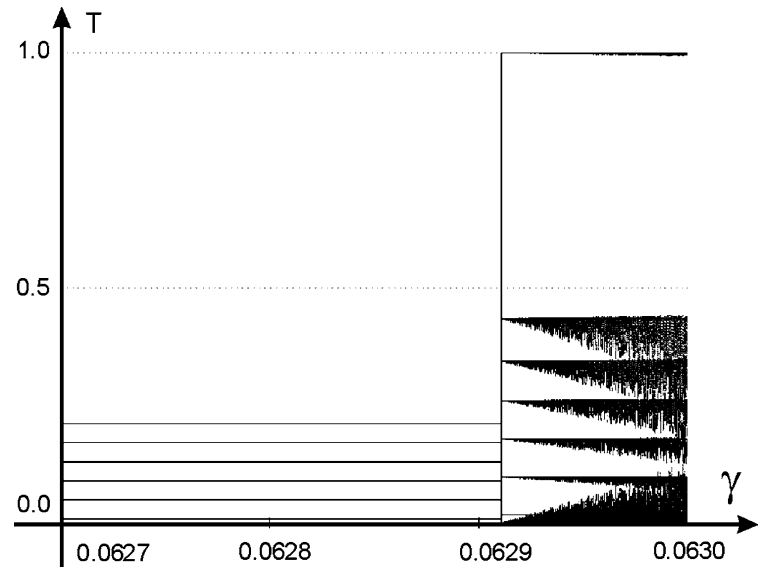

Fig. 3 Bifurcation diagram, $R=0.85, \gamma \in[0.0627,0.0630]$. Vertical critical line marks end of stability of fixed points and birth of chaotic dynamics

$$
\begin{array}{ll}
T_{0}+\frac{1-R^{n}}{(1-16 \gamma)(1-R)} v_{0} \leq \frac{1}{2}, & T_{0} \in\left[0, \frac{1}{2}\right], \\
T_{0}+\frac{1-R^{n}}{(1+16 \gamma)(1-R)} v_{0} \leq 1, & T_{0} \in\left[\frac{1}{2}, 1\right],
\end{array}
$$

and conditions for grazing are obtained for $n \rightarrow \infty$, $R^{n} \rightarrow 0$.

\subsection{Chaotic bands and homoclinic structure}

Magnification of Fig. 2 near the origin of chaotic bands is shown in Fig. 3.

There are six chaotic bands (and six basins of attraction) above the critical point $\gamma_{\mathrm{cr}}$. The first band which appears for appropriate initial conditions is shown in Fig. 4. Each band consists of two subbands only since due to cyclic periodic conditions points $T=0, T=1$ are identified.

We note that there is a switch of stability-fixed points become unstable precisely at $\gamma=\gamma_{\mathrm{cr}}$ when chaotic bands are born. Then at $\gamma=\gamma_{\mathrm{cr}}$, a homoclinic trajectory with $T=1^{-}$is probably created-this is suggested by presence of clusters of points near $T=0$, $T=1$.

Computer simulations show that just below $\gamma_{\mathrm{cr}}$ there are six unstable 2-cycles. For $\gamma<\gamma_{\text {cr }}$ impacts occur in the following time intervals: $T_{* 1} \in\left(0, \frac{1}{2}\right)$, $T_{* 2} \in\left(1+m, 1 \frac{1}{2}+m\right), \quad T_{* 3} \in\left(2+n, 2+n+\frac{1}{2}\right)$, $m=0,1,2, \ldots, n=m+2$ and $T_{* 3}=T_{* 1} \bmod 1$ where true impact times without cyclic conditions 
are shown. Then at $\gamma=\gamma_{\mathrm{cr}}$, a corner event occurs, i.e., $T_{* 2}=1$-indeed, at $T_{* 2}=1$ acceleration of the table is discontinuous. Then, for $\gamma>\gamma_{\mathrm{cr}}$, another six unstable 2-cycles are created with impact times $T_{* 1} \in\left(0, \frac{1}{2}\right), T_{* 2} \in\left(\frac{1}{2}+m, 1+m\right), T_{* 3} \in(2+n$, $\left.2+n+\frac{1}{2}\right), m=0,1,2, \ldots, n=m+2$ and $T_{* 3}=$ $T_{* 1} \bmod 1$.

Critical 2-cycle with $m=0, n=2(R=0.85)$ is shown in Fig. 5 below. This is indeed a corner eventthe time of second impact occurs at $T_{* 2}=1$ when acceleration of the table is discontinuous.

It is now possible to write down equations for the unstable 2-cycles for $\gamma \leq \gamma_{\mathrm{cr}}$, suggested by numerical

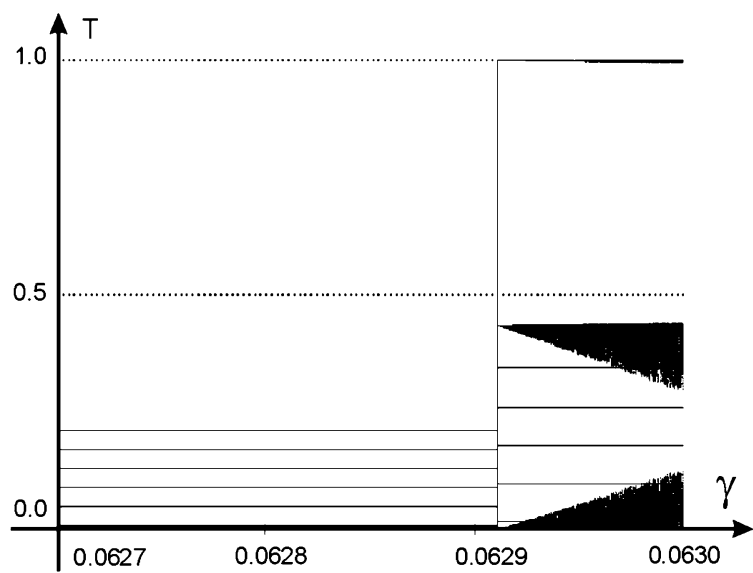

Fig. 4 Bifurcation diagram. First chaotic band, $R=0.85$, $\gamma \in[0.0627,0.0630]$. Horizontal solid lines marks positions of the fixed points to the left of the critical vertical line and positions of other chaotic bands to the right of the critical line computations discussed above:

$$
\left\{\begin{aligned}
16 \gamma & \left(y_{m}-\frac{1}{2}\right)\left(y_{m}-1\right) \\
= & -16 \gamma x\left(x-\frac{1}{2}\right)-(y-x)^{2}+(y-x) u \\
v & =-R u+2 R(y-x)+\gamma(1+R)\left(32 y_{m}-24\right), \\
-16 \gamma z_{n}\left(z_{n}-\frac{1}{2}\right) & \\
= & 16 \gamma\left(y_{m}-\frac{1}{2}\right)\left(y_{m}-1\right) \\
& -\left(z-y_{m}\right)^{2}+\left(z-y_{m}\right) v \\
w= & -R v+2 R\left(z-y_{m}\right)+\gamma(1+R)\left(8-32 z_{n}\right), \\
z_{n}= & x \\
w= & u
\end{aligned}\right.
$$

where

$$
\left\{\begin{array}{l}
x=T_{* 1}, \quad y=T_{* 2}, \quad z=T_{* 3}, \\
u=V_{* 1}, \quad v=V_{* 2}, \quad w=V_{* 3}, \\
y_{m}=T_{* 2}-m, \quad z_{n}=T_{* 3}-n,
\end{array}\right.
$$

with integer $m, n$ and $n=m+2$.

We are going to solve (23), (24) at $\gamma=\gamma_{\mathrm{cr}}$ and this means that we have to put $y_{m}=1$. It follows that there are six unstable 2-cycles in question which are obtained for $m=0,1, \ldots, 5$ and $n=m+2$, solutions for larger $m$ 's being nonphysical. Solving these equations,
Fig. 5 Displacement of the table $Y_{q}(T)$-solid line, and critical 2-cycle $\left(T_{* 2}=1\right)$-open circles

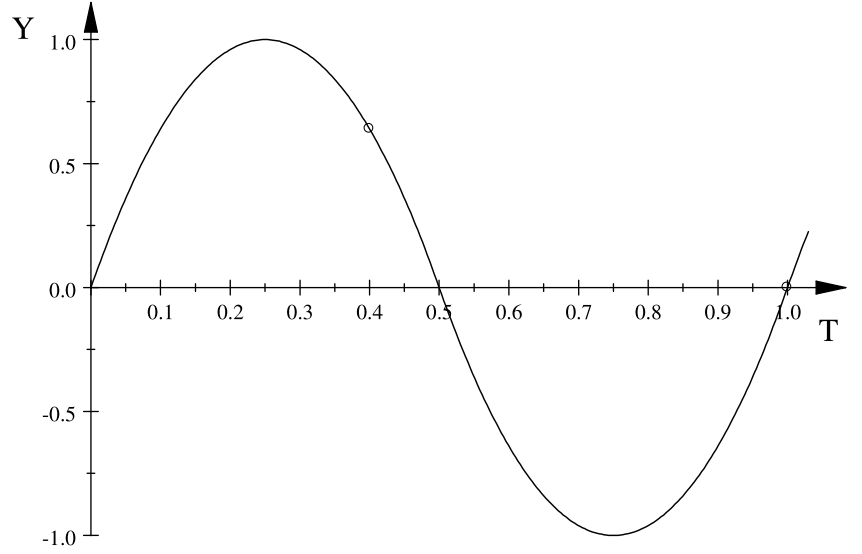


Table 1 Initial conditions for chaotic bands

\begin{tabular}{lllllll}
\hline$m$ & 0 & 1 & 2 & 3 & 4 & 5 \\
\hline$T_{* 1, m}^{(\mathrm{cr})}$ & 0.4347 & 0.3436 & 0.2602 & 0.1784 & 0.0974 & 0.0167 \\
$V_{* 1, m}^{(\mathrm{cr})}$ & 0.5148 & 1.6237 & 2.7169 & 3.8065 & 4.8946 & 5.9819 \\
$T_{* 2, m}^{(\mathrm{cr})}$ & $1^{-}$ & $1^{-}$ & $1^{-}$ & $1^{-}$ & $1^{-}$ & $1^{-}$ \\
$V_{* 2, m}^{(\mathrm{cr})}$ & 1.4546 & 2.3667 & 3.2794 & 4.1923 & 5.1051 & 6.0180 \\
\hline
\end{tabular}

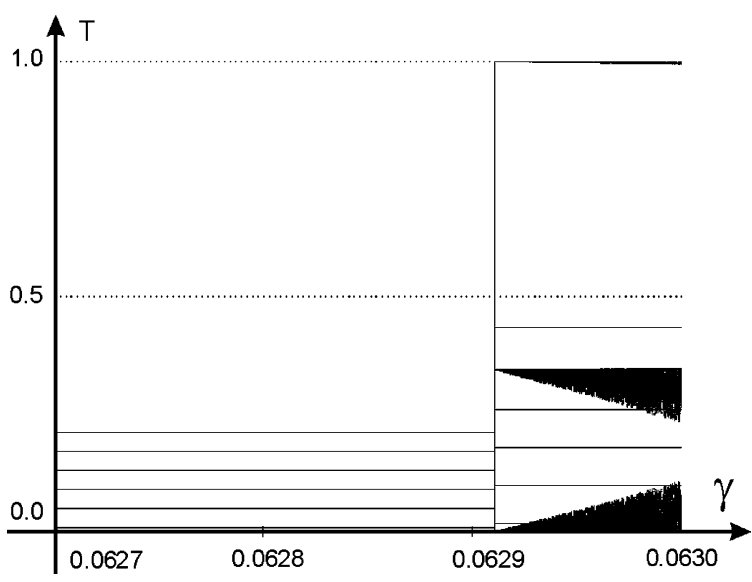

Fig. 6 Bifurcation diagram. Second chaotic band, $R=0.85$, $\gamma \in[0.0627,0.0630]$. Horizontal solid lines marks positions of the fixed points to the left of the critical vertical line and positions of other chaotic bands to the right of the critical line

we get:

$$
\begin{aligned}
& \gamma_{\mathrm{cr}}=\frac{1+R^{2}}{8(1+R)^{2}}, \quad n=m+2, \\
& T_{* 1, m}^{(\mathrm{cr})} \equiv x=\frac{2 R^{2}-6-4 m}{2\left(R^{2}-1\right)} \\
&+\frac{2 \sqrt{\left(3+5 m+2 m^{2}\right) R^{4}+1+3 m+2 m^{2}}}{2\left(R^{2}-1\right)} \\
&-m-2,
\end{aligned}
$$

and we do not show more complicated expressions for $T_{* 2, m}^{(\mathrm{cr})}, V_{* 1, m}^{(\mathrm{cr})}, V_{* 2, m}^{(\mathrm{cr})}$.

It follows from (25) that for $R=0.85$ there are only six acceptable solutions with $T_{* 1, m}^{(\mathrm{cr})}>0$ corresponding to six chaotic bands in Fig. 3.

In Table 1 impact times and the corresponding velocities just after the impact, computed from (23), (25) are listed for $R=0.85$ and $m=0,1, \ldots, 5$.

In Fig. 6, the second chaotic band is shown.
Table 2 Lyapunov exponents at the onset of chaotic bands

\begin{tabular}{lllllll}
\hline$m$ & 0 & 1 & 2 & 3 & 4 & 5 \\
\hline$\lambda_{m}$ & 0.15 & 0.145 & 0.145 & 0.15 & 0.15 & 0.18 \\
\hline
\end{tabular}

The bifurcation diagrams in Figs. 3, 4, 6 were computed in the case of $\gamma>\gamma_{\mathrm{cr}}=0.06291 \ldots$ for initial conditions shown in the Table 1 . Sharp edges of chaotic bands are given within good approximation by $T_{* 1, m}^{\text {(cr) }}$, see horizontal lines in Figs. 3, 4, 6. It seems that the homoclinic structure exists for all values of $\gamma>\gamma_{\mathrm{cr}}$ shown in the figures.

We have also computed Lyapunov exponents for $\gamma$ slightly greater than $\gamma_{\mathrm{cr}}$, cf. Table 2 .

It follows that all values of Lyapunov exponents are very similar with $\lambda_{5}$ carrying the largest computational error. The Lyapunov exponents are positive - this confirms chaotic dynamics in the bands.

\section{Discussion and closing remarks}

We have studied dynamics of a material point moving vertically in a gravitational field and colliding with a limiter. Displacement of the limiter has been assumed as quadratic and periodic function of time (6). Due to the simplicity of the problem, it was possible to investigate the dynamics analytically with some support from numerical computations. Firstly, fixed points were found and their stability was determined. Secondly, equations for a stable 2-cycle were found and simplified, cf. (14), (15). From these equations, the analytical condition for birth of the 2-cycle was found (cf. $\gamma=\gamma_{3}$ in (16)). We have also provided analytical conditions for $n$ impacts in the subinterval $0 \leq T<\frac{1}{2}$ or $\frac{1}{2} \leq T \leq 1$ and for grazing. Finally, a transition to chaotic dynamics was described in analytical terms. It was shown that six stable chaotic bands appear from six unstable 2-cycles. Equations for these 2-cycles were found and solved to yield critical value of $\gamma$, impact times and the corresponding velocities at $\gamma=\gamma_{\mathrm{cr}}$; see (25) where $\gamma_{\mathrm{cr}}$ and $T_{* 1, m}^{\text {(cr) }}$ were given. Approximation to the band edges was also found.

We have demonstrated, combining analytical and numerical approach, that at the transition point $\gamma=\gamma_{\mathrm{cr}}$ unstable 2-cycles give rise to homoclinic structures which lead to chaotic behaviour. This transition is a corner-type bifurcation similar to that found in a 
bouncing ball model with piecewise linear velocity [15]. In our future work, we shall study models with displacement of the table described by cubic functions of time [16].

Open Access This article is distributed under the terms of the Creative Commons Attribution Noncommercial License which permits any noncommercial use, distribution, and reproduction in any medium, provided the original author(s) and source are credited.

\section{References}

1. di Bernardo, M., Budd, C.J., Champneys, A.R., Kowalczyk, P.: Piecewise-Smooth Dynamical Systems. Theory and Applications. Applied Mathematical Sciences, vol. 163. Springer, Berlin (2008)

2. Luo, A.C.J.: Singularity and Dynamics on Discontinuous Vector Fields. Monograph Series on Nonlinear Science and Complexity, vol. 3. Amsterdam, Elsevier (2006)

3. Awrejcewicz, J., Lamarque, C.-H.: Bifurcation and Chaos in Nonsmooth Mechanical Systems. World Scientific Series on Nonlinear Science: Series A, vol. 45. World Scientific, Singapore (2003)

4. Filippov, A.F.: Differential Equations with Discontinuous Right-Hand Sides. Kluwer Academic, Dordrecht (1988)

5. Stronge, W.J.: Impact Mechanics. Cambridge University Press, Cambridge (2000)

6. Mehta, A. (ed.): Granular Matter: An Interdisciplinary Approach. Springer, Berlin (1994)

7. Knudsen, C., Feldberg, R., True, H.: Bifurcations and chaos in a model of a rolling wheel-set. Philos. Trans. R. Soc. Lond. A 338, 455-469 (1992)

8. Wiercigroch, M., Krivtsov, A.M., Wojewoda, J.: Vibrational energy transfer via modulated impacts for percussive drilling. J. Theor. Appl. Mech. 46, 715-726 (2008)
9. Luo, A.C.J., Guo, Y.: Motion switching and chaos of a particle in a generalized Fermi-acceleration oscillator. In: Mathematical Problems in Engineering. vol. 2009 (2009). Article ID 298906, 40 pages. doi:10.1155/2009/298906

10. Giusepponi, S., Marchesoni, F.: The chattering dynamics of an ideal bouncing ball. Europhys. Lett. 64, 36 (2003)

11. Giusepponi, S., Marchesoni, F., Borromeo, M.: Randomness in the bouncing ball dynamics. Physica A 351, 142158 (2005)

12. Okninski, A., Radziszewski, B.: Dynamics of impacts with a table moving with piecewise constant velocity. In: Cempel, C., Dobry, M.W. (eds.) Vibrations in Physical Systems, Poznań, vol. XXIII, pp. 289-294 (2008)

13. Okninski, A., Radziszewski, B.: Dynamics of a material point colliding with a limiter moving with piecewise constant velocity. In: Awrejcewicz, J. (ed.) Modelling, Simulation and Control of Nonlinear Engineering Dynamical Systems. State-of-the Art, Perspectives and Applications, pp. 117-127. Springer, Berlin (2009)

14. Okninski, A., Radziszewski, B.: Dynamics of impacts with a table moving with piecewise constant velocity. Nonlinear Dyn. 58, 515-523 (2009)

15. Okninski, A., Radziszewski, B.: Chaotic dynamics in a simple bouncing ball model. Acta Mech. Sin. 27, 130-134 (2011). arXiv:1002.2448 [nlin.CD]

16. Okninski, A., Radziszewski, B.: Simple models of bouncing ball dynamics and their comparison (2010). arXiv:1006.1236 [nlin.CD]

17. Okninski, A., Radziszewski, B.: Grazing dynamics and dependence on initial conditions in certain systems with impacts (2007). arXiv:0706.0257 [nlin.CD]

18. Luo, A.C.J., Han, R.P.S.: The dynamics of a bouncing ball with a sinusoidally vibrating table revisited. Nonlinear Dyn. 10, 1-18 (1996)

19. Luo, A.C.J.: Discontinuous Dynamical System on Timevarying Domains. Nonlinear Physical Science. Higher Education Press, Beijing and Springer, Dordrecht, Heidelberg, London, New York (2009) 